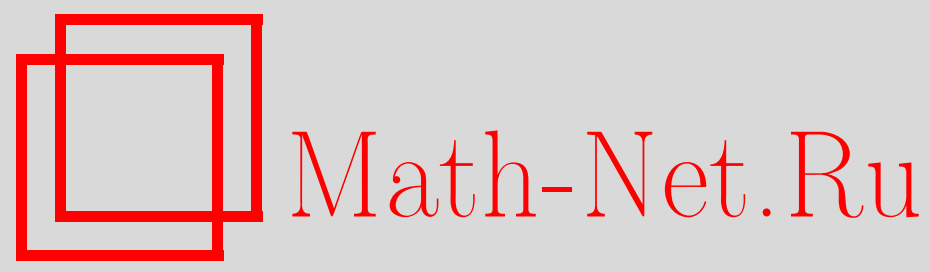

Н. М. Добровольский, А. Л. Рощеня, О числе точек решетки в гиперболическом кресте, Матем. заметки, 1998, том 63, выпуск 3, 363-369

DOI: https://doi.org/10.4213/mzm1290

Использование Общероссийского математического портала Math-Net.Ru подразумевает, что вы прочитали и согласны с пользовательским соглашением http://www . mathnet.ru/rus/agreement

Параметры загрузки:

IP: 54.198 .64 .247

26 апреля 2023 г., 14:16:21

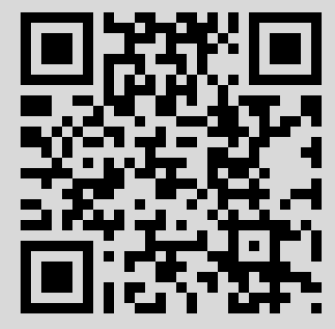


УДК 511.9

\title{
О ЧИСЛЕ ТОЧЕК РЕШЕТКИ В ГИПЕРБОЛИЧЕСКОМ КРЕСТЕ
}

\author{
Н. М. Добровольский, А. Л. Рощеня
}

В работе получена асимптотическая формула для числа точек произвольной решетки в гиперболическом кресте.

Библиография: 2 названия.

Пусть $s \geqslant 2$ и $\Lambda \subset \mathbb{R}^{s}$ - полная решетка. Гиперболическим крестом называется область $K(T)=\left\{\vec{x} \mid \bar{x}_{1} \cdots \bar{x}_{s} \leqslant T\right\}$, где для вещественного $x$ обозначаем $\bar{x}=\max (1,|x|)$. Пусть $D(T \mid \Lambda)$ - количество ненулевых точек решетки $\Lambda$ в гиперболическом кресте $K(T)$. Цель данной работы - доказать следующую теорему.

ТЕОремА. Для любой решетки $\Lambda$ при $T \geqslant 3$ справедливо асимптотическое равенство

$$
D(T \mid \Lambda)=\frac{2^{s} T \ln ^{s-1} T}{(s-1) ! \operatorname{det} \Lambda}+\Theta C(\Lambda) \frac{T \ln ^{s-2} T}{\operatorname{det} \Lambda},
$$

әде $C(\Lambda)=2^{s} e\left(a_{0}+2\right)^{s} u|\Theta| \leqslant 1$.

1. Обозначения. Пусть $\vec{\lambda}_{j}=\left(\lambda_{j 1}, \ldots, \lambda_{j s}\right)(j=1, \ldots, s)$ - произвольньй фиксированный базис решетки $\Lambda$ и

$$
A=A\left(\vec{\lambda}_{1}, \ldots, \vec{\lambda}_{s}\right)=\max _{1 \leqslant j \leqslant s} \frac{1}{2} \sum_{\nu=1}^{s}\left|\lambda_{\nu j}\right|
$$

$\vec{\lambda}_{j}^{*}=\left(\lambda_{j 1}^{*}, \ldots, \lambda_{j s}^{*}\right)(j=1, \ldots, s)$ - взаимный базис взаимной решетки $\Lambda^{*}$ (как известно [1], взаимньй базис задается соотношениями

$$
\left(\vec{\lambda}_{i}, \vec{\lambda}_{j}^{*}\right)=\sum_{\nu=1}^{s} \lambda_{i \nu} \lambda_{j \nu}^{*}=\delta_{i j}= \begin{cases}1 & \text { при } i=j \\ 0 & \text { при } i \neq j\end{cases}
$$

а взаимная решетка $\Lambda^{*}$ однозначно определяется решеткой $\Lambda$ ).

Определим следующие области:

$$
\Pi(T \mid \Lambda)=\left\{\vec{t}: \prod_{j=1}^{s} \overline{\sum_{\nu=1}^{s} \lambda_{\nu j} t_{\nu}+\sum_{\nu=1}^{s} \lambda_{\nu j}\left(\frac{1}{2}-\left\{t_{\nu}+\frac{1}{2}\right\}\right)} \leqslant T\right\}
$$


для целого вектора $\vec{m}$

$$
\begin{gathered}
\Pi(\vec{m})=\left\{\vec{t}:\left[t_{\nu}+\frac{1}{2}\right]=m_{\nu}, \nu=1, \ldots, s\right\} \\
\Pi^{*}(T \mid \Lambda)=\left\{\vec{y}: \prod_{j=1}^{s} y_{j}+\sum_{\nu=1}^{s} \lambda_{\nu j}\left(\frac{1}{2}-\left\{\frac{1}{2}+\sum_{k=1}^{s} y_{k} \lambda_{\nu k}^{*}\right\}\right) \leqslant T\right\}
\end{gathered}
$$

при $a \geqslant 0$

$$
\Pi_{1}(T, a)=\left\{\vec{y}: \prod_{j=1}^{s} \overline{\left|y_{j}\right|+a} \leqslant T\right\}, \quad \Pi_{2}(T, a)=\left\{\vec{y}: \prod_{j=1}^{s} u\left(y_{j}, a\right) \leqslant T\right\},
$$

где

$$
u(y, a)= \begin{cases}1 & \text { при }|y| \leqslant a+1, \\ |y|-a & \text { при }|y| \geqslant a+1 .\end{cases}
$$

Заметим, что $\Pi_{1}(T, 0)=\Pi_{2}(T, 0)=K(T)$. Пусть при $a \geqslant 0, T \geqslant 0$

$$
I_{s}(a, T)=\int_{\substack{\prod_{j=1}^{s}\left(y_{j}+a\right) \leqslant T \\ y_{1}, \ldots, y_{s} \geqslant 0}} d \vec{y}, \quad J_{s}(a, T)=\int_{\substack{\prod_{j=1}^{s} u\left(y_{j}, a\right) \leqslant T \\ y_{1}, \ldots, y_{s} \geqslant 0}} d \vec{y} .
$$

\section{2. Вспомогательные леммы.}

ЛЕмма 1. Справедливо равенство

$$
\sum_{\Pi_{j=1}^{s}} \int_{\Pi(\vec{m})} d \vec{t}=\int_{\Pi(T \mid \Lambda)} d \vec{t}
$$

ДокАЗАТЕЛЬСТво. Для любых целых неравных векторов $\vec{n}$ и $\vec{m}$ области $\Pi(\vec{n})$ и $\Pi(\vec{m})$ не пересекаются. С другой стороны, если $m_{\nu}=\left[t_{\nu}+1 / 2\right](\nu=1, \ldots, s)$, то

$$
\sum_{\nu=1}^{s} \lambda_{\nu j} t_{\nu}+\sum_{\nu=1}^{s} \lambda_{\nu j}\left(\frac{1}{2}-\left\{t_{\nu}+\frac{1}{2}\right\}\right)=\sum_{\nu=1}^{s} \lambda_{\nu j} m_{\nu}
$$

и, значит,

$$
\Pi(T \mid \Lambda)=\bigcup_{\prod_{j=1}^{s} \frac{\bigcup}{\lambda_{1 j} m_{1}+\cdots+\lambda_{s j} m_{s}} \leqslant T} \Pi(\vec{m}) .
$$

Отсюда в силу аддитивности интеграла следует утверждение леммы.

ЛЕмма 2. Справедливы равенства

$$
\begin{gathered}
\int_{\Pi(T \mid \Lambda)} d \vec{t}=\frac{1}{\operatorname{det} \Lambda} \int_{\Pi^{*}(T \mid \Lambda)} d \vec{y} \\
\int_{\Pi_{1}(T, a)} d \vec{y}=2^{s} I_{s}(a, T) \quad n p u \quad a \geqslant 1, \\
\int_{\Pi_{2}(T, a)} d \vec{y}=2^{s} J_{s}(a, T) \quad n p u \quad a \geqslant 0 .
\end{gathered}
$$


ДоКАЗАТЕЛЬСТво. Положим

$$
\vec{y}=\left(y_{1}, \ldots, y_{s}\right)=t_{1} \vec{\lambda}_{1}+\cdots+t_{s} \vec{\lambda}_{s}
$$

Тогда

$$
y_{\nu}=\sum_{j=1}^{s} t_{j} \lambda_{j \nu} \quad(\nu=1, \ldots, s), \quad t_{j}=\left(\vec{y}, \vec{\lambda}_{j}^{*}\right)=\sum_{\nu=1}^{s} y_{\nu} \lambda_{j \nu}^{*} .
$$

Сделав замену переменных (5), получим $d \vec{t}=(\operatorname{det} \Lambda)^{-1} d \vec{y}$,

$$
\begin{aligned}
\int_{\Pi(T \mid \Lambda)} d \vec{t} & =\frac{1}{\operatorname{det} \Lambda} \int_{\prod_{j=1}^{s} \frac{1}{y_{j}+\sum_{\nu=1}^{s} \lambda_{\nu j}\left(1 / 2-\left\{1 / 2+\sum_{k=1}^{s} y_{k} \lambda_{\nu k}^{*}\right\}\right) \leqslant T}} d \vec{y} \\
& =\frac{1}{\operatorname{det} \Lambda} \int_{\Pi^{*}(T \mid \Lambda)} d \vec{y},
\end{aligned}
$$

и равенство (2) доказано.

При $a \geqslant 1$ имеем $|y|+a=\overline{|y|+a}$ и, следовательно, в силу симметрии области $\Pi_{1}(T, a)$ имеем

$$
\int_{\Pi_{1}(T, a)} d \vec{y}=2^{s} \int_{\substack{\prod_{j=1}^{s}\left(y_{j}+a\right) \leqslant T \\ y_{1}, \ldots, y_{s} \geqslant 0}} d \vec{y}=2^{s} I_{s}(a, T),
$$

что доказывает равенство (3). Наконец, равенство (4) следует из симметрии области $\Pi_{2}(T, a)$ при любом $a \geqslant 0$.

Лемма 3. При $а>0, T \geqslant a^{s}$ справедливо равенство

$$
I_{s}(a, T)=(-1)^{s+1}\left(T-a^{s}\right)+T \sum_{n=1}^{s-1} \frac{(\ln T-s \ln a)^{n}(-1)^{s-1-n}}{n !} .
$$

ДокАЗАТЕльСтво. Прежде всего установим для $I_{s}(a, T)$ рекуррентное соотношение. Имеем

$$
\begin{aligned}
I_{s}(a, T) & =\int_{0}^{T / a^{s-1}-a} d y_{s} \int_{\substack{\prod_{j=1}^{s-1}\left(y_{j}+a\right) \leqslant \frac{T}{y_{s}+a} \\
y_{1}, \ldots, y_{s-1} \geqslant 0}} d y_{1} \cdots d y_{s-1} \\
& =\int_{0}^{T / a^{s-1}-a} I_{s-1}\left(a, \frac{T}{y+a}\right) d y=\int_{a}^{T / a^{s-1}} I_{s-1}\left(a, \frac{T}{y}\right) d y .
\end{aligned}
$$

Далее проведем индукцию по $s$, используя (7). При $s=1$ имеем

$$
I_{1}(a, T)=\int_{0}^{T-a} d y=T-a
$$

и равенство (6) вьполнено. Из (7) и предположения индукции для $s-1$ получим

$$
\begin{aligned}
I_{s}(a, T) & =\int_{a}^{T / a^{s-1}}\left((-1)^{s}\left(\frac{T}{y}-a^{s-1}\right)\right. \\
+ & \left.\frac{T}{y} \sum_{n=1}^{s-2} \frac{(\ln (T / y)-(s-1) \ln a)^{n}(-1)^{s-2-n}}{n !}\right) d y
\end{aligned}
$$




$$
\begin{aligned}
= & (-1)^{s} T(\ln T-(s-1) \ln a-\ln a)+(-1)^{s+1} a^{s-1}\left(\frac{T}{a^{s-1}}-a\right) \\
& +T \int_{\ln a}^{\ln T-(s-1) \ln a} \sum_{n=1}^{s-2} \frac{(\ln T-u-(s-1) \ln a)^{n}(-1)^{s-2-n}}{n !} d u \\
= & (-1)^{s+1}\left(T-a^{s}\right)+(-1)^{s} T(\ln T-s \ln a)+T \sum_{n=1}^{s-2} \frac{(-1)^{s-2-n}}{n !} \int_{0}^{\ln T-s \ln a} t^{n} d t \\
= & (-1)^{s+1}\left(T-a^{s}\right)+T \sum_{n=1}^{s-2} \frac{(-1)^{s-2-n}}{n !} \frac{(\ln T-s \ln a)^{n+1}}{n+1}+(-1)^{s} T(\ln T-s \ln a) \\
= & (-1)^{s+1}\left(T-a^{s}\right)+T \sum_{n=1}^{s-1} \frac{(-1)^{s-1-n}(\ln T-s \ln a)^{n}}{n !},
\end{aligned}
$$

что и доказьвает утверждение леммы.

Лемма 4. При $a \geqslant 0, T \geqslant 1, s \geqslant 1$ справедливо равенство

$$
J_{s}(a, T)=a^{s}+\sum_{n=0}^{s-1} \frac{T \ln ^{n} T}{n !} \sum_{k=0}^{s-1-n} C_{s}^{k} C_{s-k-1}^{n} a^{k}
$$

ДокАЗАТЕЛЬСТво. Из определения $J_{s}(a, T)$ имеем

$$
\begin{aligned}
J_{s}(a, T)= & \int_{0}^{a+1} d y_{s} \int_{\begin{array}{c}
y_{1}, \ldots, y_{s-1} \geqslant 0 \\
\left.y_{1}, a\right) \cdots u\left(y_{s-1}, a\right) \leqslant T
\end{array}} d y_{1} \cdots d y_{s-1} \\
& +\int_{a+1}^{T+a} d y_{s} \int_{\begin{array}{c}
u\left(y_{1}, a\right) \cdots u\left(y_{s-1}, a\right) \leqslant T /(y-a) \\
y_{1}, \ldots, y_{s-1} \geqslant 0
\end{array}} d y_{1} \cdots d y_{s-1} \\
= & (a+1) J_{s-1}(a, T)+\int_{1}^{T} J_{s-1}\left(a, \frac{T}{y}\right) d y
\end{aligned}
$$

Далее проведем индукцию по $s$, используя рекуррентное равенство (9). При $s=1$ имеем

$$
J_{1}(a, T)=\int_{u(y, a) \leqslant T}^{y \geqslant 0} d y=\int_{0}^{a+1} d y+\int_{a+1}^{a+T} d y=a+1+T-1=a+T
$$

и равенство (8) вьполнено.

Пусть

$$
Q_{s, n}(a)=\sum_{k=0}^{s-1-n} C_{s}^{k} C_{s-k-1}^{n} a^{k}, \quad J_{s}(a, T)=a^{s}+\sum_{n=0}^{s-1} \frac{T \ln ^{n} T}{n !} Q_{s, n}(a) .
$$


Тогда

$$
\begin{aligned}
J_{s+1}(a, T)=(a+1) J_{s}(a, T)+\int_{1}^{T} J_{s}\left(a, \frac{T}{y}\right) d y \\
=(a+1) a^{s}+\sum_{n=0}^{s-1} \frac{T \ln ^{n} T}{n !} Q_{s, n}(a)(a+1)+\int_{1}^{T}\left(a^{s}+\sum_{n=0}^{s-1} \frac{T / y \cdot \ln ^{n}(T / y)}{n !} Q_{s, n}(a)\right) d y \\
=a^{s+1}+a^{s}+T a^{s}-a^{s}+\sum_{n=0}^{s-1} \frac{T \ln ^{n} T}{n !} Q_{s, n}(a)(a+1)+\sum_{n=0}^{s-1} \frac{T}{n !} Q_{s, n}(a) \frac{\ln ^{n+1} T}{n+1} \\
=a^{s+1}+T\left(a^{s}+Q_{s, 0}(a)(a+1)\right) \\
\quad+\sum_{n=1}^{s-1} \frac{T \ln ^{n} T}{n !}\left(Q_{s, n}(a)(a+1)+Q_{s, n-1}(a)\right)+\frac{T \ln ^{s} T}{s !} Q_{s, s-1}(a) \\
=a^{s+1}+\sum_{n=0}^{s} \frac{T \ln ^{n} T}{n !} Q_{s+1, n}(a),
\end{aligned}
$$

где

$$
\begin{aligned}
Q_{s+1,0}(a) & =a^{s}+(a+1) \sum_{k=0}^{s-1} C_{s}^{k} a^{k}=\sum_{k=1}^{s} C_{s}^{k-1} a^{k}+\sum_{k=0}^{s-1} C_{s}^{k} a^{k}+a^{s} \\
& =s a^{s}+\sum_{k=1}^{s-1}\left(C_{s}^{k-1}+C_{s}^{k}\right) a^{k}+1=\sum_{k=0}^{s} C_{s+1}^{k} a^{k}=\sum_{k=0}^{s-1-0} C_{s+1}^{k} C_{s+1-k-1}^{0} a^{k}, \\
Q_{s+1, s}(a) & =Q_{s, s-1}(a)=1=\sum_{k=0}^{(s+1)-1-s} C_{s+1}^{k} C_{s+1-k-1}^{s} a^{k},
\end{aligned}
$$

и при $1 \leqslant n \leqslant s-1$

$$
\begin{aligned}
Q_{s+1, n}(a)=Q_{s, n}(a)(a+1)+Q_{s, n-1}(a) \\
=(a+1) \sum_{k=0}^{s-1-n} C_{s}^{k} C_{s-k-1}^{n} a^{k}+\sum_{k=0}^{s-1-(n-1)} C_{s}^{k} C_{s-k-1}^{n-1} a^{k} \\
=\sum_{k=1}^{s-n} C_{s}^{k-1} C_{s-(k-1)-1}^{n} a^{k}+\sum_{k=0}^{s-1-n} C_{s}^{k}\left(C_{s-k-1}^{n}+C_{s-k-1}^{n-1}\right) a^{k}+C_{s}^{s-n} C_{n-1}^{n-1} a^{s-n} \\
=\sum_{k=1}^{s-n} C_{s}^{k-1} C_{s-k}^{n} a^{k}+\sum_{k=0}^{s-n} C_{s}^{k} C_{s-k}^{n} a^{k}=\sum_{k=1}^{s-n}\left(C_{s}^{k-1}+C_{s}^{k}\right) C_{s-k}^{n} a^{k}+C_{s}^{0} C_{s-0}^{n} a^{0} \\
=\sum_{k=0}^{s-n}\left(C_{s+1}^{k} C_{(s+1)-1-k}^{n}\right) a^{k}
\end{aligned}
$$

и, значит, $J_{s+1}(a, T)$ удовлетворяет равенству (8), что доказывает лемму.

СлЕДСтвиЕ 1. При $а>1, T \geqslant 3$ справедливо неравенство

$$
I_{s}(a, T) \geqslant \frac{T \ln ^{s-1} T}{(s-1) !}-e a^{s} T \ln ^{s-2} T-a^{s} .
$$


ДокАЗАТЕЛЬСтво. Действительно,

$$
\begin{aligned}
I_{s}(a, T)= & (-1)^{s+1}\left(T-a^{s}\right)+T \sum_{n=1}^{s-1} \frac{(\ln T-s \ln a)^{n}(-1)^{s-1-n}}{n !} \\
= & T \sum_{n=1}^{s-1} \frac{(-1)^{s-1-n}}{n !} \sum_{k=0}^{n} C_{n}^{k} \ln ^{k} T(-1)^{n-k}(s \ln a)^{n-k}+(-1)^{s+1} T+(-1)^{s} a^{s} \\
= & (-1)^{s} a^{s}+T \sum_{k=1}^{s-1} \ln ^{k} T \sum_{n=k}^{s-1} \frac{(-1)^{s-1-n}(-1)^{n-k}}{n !} C_{n}^{k}(s \ln a)^{n-k} \\
& +T\left((-1)^{s+1}+\sum_{n=1}^{s-1} \frac{(-1)^{s-1-n}}{n !}(-1)^{n}(s \ln a)^{n}\right) \\
= & (-1)^{s} a^{s}+(-1)^{s+1} T \sum_{n=0}^{s-1} \frac{(s \ln a)^{n}}{n !}+T \sum_{k=1}^{s-1} \ln ^{k} T(-1)^{s-1-k} \sum_{n=0}^{s-k-1} \frac{(s \ln a)^{n}}{k ! n !} \\
= & \frac{T \ln ^{s-1} T}{(s-1) !}+T \sum_{k=0}^{s-2} \frac{\ln k T(-1)^{s-1-k}}{k !} \sum_{n=0}^{s-k-1} \frac{(s \ln a)^{n}}{n !}+(-1)^{s} a^{s} \\
\geqslant & \frac{T \ln ^{s-1} T}{(s-1) !}-T \ln ^{s-2} T \sum_{k=0}^{s-2} \frac{1}{k !} \sum_{n=0}^{s-k-1} \frac{(s \ln a)^{n}}{n !}-a^{s} \\
\geqslant & \frac{T \ln ^{s-1} T}{(s-1) !}-T \ln ^{s-2} T \sum_{k=0}^{s-1} \frac{1}{k !} a^{s}-a^{s} \geqslant \frac{T \ln ^{s-1} T}{(s-1) !}-e a^{s} T \ln ^{s-2} T-a^{s},
\end{aligned}
$$

что и требовалось доказать.

СлЕДСтвиЕ 2. Справедливо неравенство

$$
J_{s}(a, T) \leqslant \frac{T \ln ^{s-1} T}{(s-1) !}+(a+2)^{s} T \ln ^{s-2} T+a^{s} .
$$

ДокАЗАТЕЛЬСтво. Действительно,

$$
\begin{aligned}
J_{s}(a, T) & =\frac{T \ln ^{s-1} T}{(s-1) !}+\sum_{n=0}^{s-2} \frac{T \ln ^{n} T}{n !} \sum_{k=0}^{s-1-n} \frac{s !(s-k-1) !}{k !(s-k) ! n !(s-k-n-1) !} a^{k}+a^{s} \\
& =\frac{T \ln ^{s-1} T}{(s-1) !}+T \sum_{n=0}^{s-2} \frac{\ln ^{n} T}{n !} C_{s}^{n} \sum_{k=0}^{s-1-n} \frac{s-n}{s-k} C_{s-n-1}^{k} a^{k}+a^{s} \\
& \leqslant \frac{T \ln ^{s-1} T}{(s-1) !}+T \sum_{n=0}^{s-2} \frac{\ln ^{n} T}{n !} C_{s}^{n} \frac{s-n}{n+1}(a+1)^{s-n-1}+a^{s} \\
& \leqslant \frac{T \ln ^{s-1} T}{(s-1) !}+(a+2)^{s} T \ln ^{s-2} T+a^{s},
\end{aligned}
$$

что и требовалось доказать. 
3. ДОКАЗАТЕЛЬСТВо ТЕОРЕМЫ. Из определения величины $D(T \mid \Lambda)$ следует, что

$$
D(T \mid \Lambda)+1=\sum_{\substack{\vec{x} \in \Lambda \\ \bar{x}_{1} \cdots \bar{x}_{s} \leqslant T}} 1=\sum_{\prod_{j=1}^{s} \bar{\lambda}_{\lambda_{j} m_{1}+\cdots+\lambda_{s j} m_{s}} \leqslant T} 1=\int_{\Pi(T \mid \Lambda)} d \vec{t}
$$

в силу леммы 1.

Применяя лемму 2 , получим

$$
D(T \mid \Lambda)+1=\frac{1}{\operatorname{det} \Lambda} \int_{\Pi^{*}(T \mid \Lambda)} d \vec{t}
$$

Пусть $a=\bar{A}$, где $A$ задано равенством (1). Тогда справедливы включения $\Pi_{1}(T, a) \subseteq$ $\Pi^{*}(T \mid \Lambda) \subseteq \Pi_{2}(T, a)$, так как

$$
\prod_{j=1}^{s} u\left(\left|y_{j}\right|, a\right) \leqslant \prod_{j=1}^{s} \overline{y_{j}+\sum_{\nu=1}^{s} \lambda_{\nu j}\left(\frac{1}{2}-\left\{\frac{1}{2}+\sum_{k=1}^{s} y_{k} \lambda_{\nu k}^{*}\right\}\right)} \leqslant \prod_{j=1}^{s}\left(\left|y_{j}\right|+a\right) .
$$

Из этого включения и леммы 2 следуют неравенства

$$
\frac{2^{s} I_{s}(a, T)}{\operatorname{det} \Lambda} \leqslant D(T \mid \Lambda)+1 \leqslant \frac{2^{s} J_{s}(a, T)}{\operatorname{det} \Lambda} .
$$

Применяя неравенства (10), (11), получим

$$
\begin{aligned}
\frac{2^{s}}{\operatorname{det} \Lambda}\left(\frac{T \ln ^{s-1} T}{(s-1) !}-e a^{s} T \ln ^{s-2} T-a^{s}\right) & \leqslant D(T \mid \Lambda)+1 \\
& \leqslant \frac{2^{s}}{\operatorname{det} \Lambda}\left(\frac{T \ln ^{s-1} T}{(s-1) !}+T \ln ^{s-2} T(a+2)^{s}+a^{s}\right) .
\end{aligned}
$$

Отсюда вытекает, что

$$
D(T \mid \Lambda)=\frac{2^{s} T \ln ^{s-1} T}{(s-1) ! \operatorname{det} \Lambda}+C(\Lambda) \Theta \frac{T \ln ^{s-2} T}{\operatorname{det} \Lambda}
$$

с $C(\Lambda)=2^{s} e\left(a_{0}+2\right)^{s}$ и $a_{0}=\min \bar{A}\left(\vec{\lambda}_{1}, \ldots, \vec{\lambda}_{s}\right)$, где минимум берется по всем базисам решетки $\Lambda$.

Теорема полностью доказана.

Основной результат настоящей статьи анонсирован в тезисе [2]. Авторы выражают благодарность профессору Н.М. Коробову и профессору В.И. Нечаеву за внимание и полезное обсуждение.

\section{СПИСОК ЦИТИРОВАННОЙ ЛИТЕРАТУРЫ}

[1] Титчмарш Э. Ч. Теория дзета-функции Римана. М., 1953.

[2] Добровольский Н. М., Рощеня А. Л. О числе точек решетки в гиперболическом кресте // II Международная конференция "Алгебраические, вероятностные, геометрические, комбинаторные и функциональные методы в теории чисел”. Тезисы докл. Воронеж: ВГУ, 1995. C. 53 . 